\title{
Surgical management of palatal teratoma (epignathus) with the use of virtual reconstruction and 3D models: a case report and literature review
}

\author{
Cynthia Minerva Gonzalez-Cantu ${ }^{1}$, Pablo Juan Moreno-Peña ${ }^{2}$, Mayela Guadalupe Salazar-Lara ${ }^{2}$, \\ Pablo Patricio Flores García ${ }^{2}$, Fernando Félix Montes-Tapia ${ }^{3}$, Victor Hugo Cervantes-Kardasch ${ }^{4}$, \\ Yanko Castro-Govea ${ }^{1}$ \\ ${ }^{1}$ Plastic, Aesthetic and Reconstructive Surgery Service, University Hospital "Dr. José Eleuterio González", Faculty of Medicine, Autonomous \\ University of Nuevo Leon, Monterrey; ${ }^{2}$ Faculty of Medicine, Autonomous University of Nuevo Leon, Monterrey; ${ }^{3}$ Pediatric Surgery Service, \\ University Hospital "Dr. José Eleuterio González", Faculty of Medicine, Autonomous University of Nuevo Leon, Monterrey; ${ }^{4}$ Laboratory of \\ Tissue Engineering, Faculty of Medicine, University of Colima, Colima, Mexico
}

\begin{abstract}
Epignathus is a rare congenital orofacial teratoma that arises from the sphenoid region of the palate or the pharynx. It occurs in approximately 1:35,000 to 1:200,000 live births representing $2 \%$ to $9 \%$ of all teratomas. We present the case of a newborn of 39.4 weeks of gestation with a tumor that occupied the entire oral cavity. The patient was delivered by cesarean section. Oral resection was managed by pediatric surgery. Plastic surgery used virtual 3-dimensional models to establish the extension, and depth of the tumor. Bloc resection and reconstruction of the epignathus were performed. The mass was diagnosed as a mature teratoma associated with cleft lip and palate, nasoethmoidal meningocele that conditions hypertelorism, and a pseudomacrostoma. Tridimensional technology was applied to plan the surgical intervention. It contributed to a better understanding of the relationships between the tumor and the adjacent structures. This optimized the surgical approach and outcome.
\end{abstract}

Keywords Teratoma / Pediatric plastic surgery / 3D reconstruction computed tomography / Improved surgical approach
Correspondence: Yanko Castro-Govea Plastic, Aesthetic and Reconstructive Surgery Service, University Hospital "Dr. José Eleuterio González", Autonomous University of Nuevo Leon, Ave. Madero y Av. Gonzalitos s/ $n$, Colonia Mitras Centro, Monterrey 64460, Mexico

Tel: $+52-81-8348-7656$

Fax: +52-81-8347-6117

E-mail: goveayanko@hotmail.com

We thank Sergio Lozano MD (Scientific Publication Support Coordinator, University Hospital "Dr. José Eleuterio González", Autonomous University of Nuevo Leon, Monterrey, Nuevo Leon, Mexico) for his review of the manuscript, and the patient, the patient's mother, and the hospital staff for their help. Also, we thank Hernan Chacon-Moreno MD (Professor of Plastic, Aesthetic and Reconstructive Surgery Service, University Hospital "Dr. José Eleuterio González", and Faculty of Medicine, Autonomous University of

Nuevo Leon, Monterrey, Nuevo Leon, Mexico) for all the facilities provided to carry out this work.

Received: February 10, 2021 - Revised: April 16, 2021 - Accepted: May 27, 2021

pISSN: 2234-6163 • elSSN: 2234-6171 • https://doi.org/10.5999/aps.2021.00318• Arch Plast Surg 2021;48:518-523

\section{INTRODUCTION}

Teratoma comes from the Greek word teratos (monster); tera- tomas are embryonic neoplasias derived from totipotent cells that contain tissue from all the germ layers (ectoderm, endoderm, and mesoderm). 
They are more frequently found in the sacrococcygeal region and are predominant in the female gender. These tumors have high mortality associated with obstruction of the airway during, or shortly after, birth [1]. Their etiology is still unknown, however, there are a variety of theories with the most popular being that the epignathus can surge from totipotent cells from Rathke's pouch and grow in a disorganized manner [2].

Teratomas can be classified into four groups: (1) dermoid cyst, which consists of epithelial cells composed of two germ cell lines (generally, ectoderm, and mesoderm); (2) teratoid cyst, which is composed of the three layers of germ cells; however, it is poorly differentiated; (3) teratomas, which can be solid or cystic, also contain tissue from the three germ layers, and are more histologically identifiable; and (4) epignathus, which is a highly developed oral tumor that originates from fetal organs and extremities. It is also known as "fetus-in-fetu" [3].

An epignathus is a rare congenital orofacial teratoma that can arise from the sphenoid region, the palate, or the pharynx and it is found in approximately $1: 35,000$ to $1: 200,000$ live births, representing $2 \%$ to $29 \%$ of all teratomas [4]. It is associated with midline malformations and a cleft palate is the most common [5].

The use of 3-dimensional (3D) printers in medicine began two decades ago. Its current technological inclusion in the field of craniofacial plastic surgery has had a great application. Its large advantage is not only millimetric surgical planning but also custom anatomical reconstruction. Another variable that provides a large benefit is that it is educational for the patient.

The great heterogeneity of teratomas, together with their particular form of presentation, causes great difficulty in defining the exact margin of resection. This logically represents a great surgical challenge in its integral management $[6,7]$.

We present the clinical case of a newborn with a complex palatine teratoma and its surgical management using virtual image reconstruction and 3D models. The clinical study was approved by the institutional review board (IRB No. CP 20-003).

\section{CASE}

A 32-year-old-woman was referred to our hospital with 39.4 weeks of gestation for evaluation by the pediatric surgery service of a female fetus with a large mixed echogenic mass located in the oropharyngeal region. The mass was initially discovered in the 36th gestational week with a 2-dimensional (2D) sonogram. The medical history revealed that the parents were healthy, nonconsanguineous, and did not have a family history of congenital anomalies.

The pediatric evaluation included 4-dimensional echography that revealed the presence of a tumor that occupied the entire oral cavity and protruded from the mouth, as well as the presence of polyhydramnios.

Due to the nature of the tumor, its anatomical location, and dimensions, the pediatric surgery service together with the gynecology and obstetrics service planned a cesarean section and an ex-utero intrapartum treatment (EXIT) procedure as a first option.

\section{Initial surgical intervention: pediatric surgery}

The large dimension of the tumor (Fig. 1) made it necessary to secure the airway at birth by tracheostomy. A female infant of 2,740 $\mathrm{g}$ was born with a height of $47 \mathrm{~cm}$, an Apgar score of 7/9, and no apparent motor or sensory alteration. On examination, mild hypertelorism was identified, as well as an incomplete right cleft lip. The tissue distension produced by the tumor caused a pseudomacrostoma of the right side. The tumor practically occupied the oral cavity and protruded from the mouth; its aspect was rough and irregular. With a tongue depressor, it was seen that the mass occupied the hard palate, also finding a fissure in the soft palate on the right side. The pediatric surgery service decided to only resect the extraoral tissue for study.

Once the newborn was stabilized after extracorporeal collection, she was hospitalized in the pediatric intensive care unit. Based on the previously mentioned characteristics and the great heterogeneity that these types of lesion represent, a consultation from the plastic surgery service was requested for integral management at a second surgical time. A pathology study reported a partially resected mature teratoma derived from the three germ layers.

During her stay in intensive care, a computed tomography

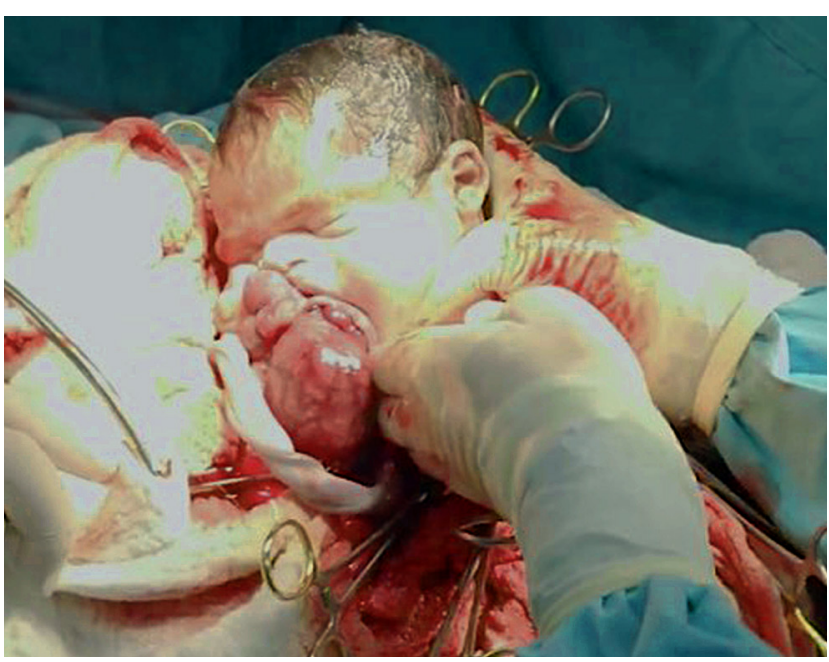

Fig. 1. Post-cesarean section image of a newborn female infant with epignathus. Appearance immediately before extraoral resection. 
(CT) scan was performed which reported that the residual tumor had irregular borders and dimensions of $0.9 \times 2.2 \times 3 \mathrm{~cm}$, predominantly occupying the hard palate, in addition to great heterogeneity in the density of its tissues. The evaluation was complemented with magnetic resonance imaging (MRI) because of the finding of polyhydramnios. In the intentional search for synchronous alterations of the central nervous system, a nasoethmoidal meningocele and the absence of the cribriform plate were identified; this conditioned the hypertelorism. The morphology and density of the brain parenchyma were conserved with adequate differentiation of the grey and white matter, grey nuclei, and normal middle-line structures.

\section{Planning of epignathus resection by virtual image reconstruction and $3 \mathrm{D}$ models}

After the assessment of the newborn with residual tumor (Fig. 2) by our plastic surgery service, we opted to plan her surgical treatment using virtual image reconstruction (3D CT) to more

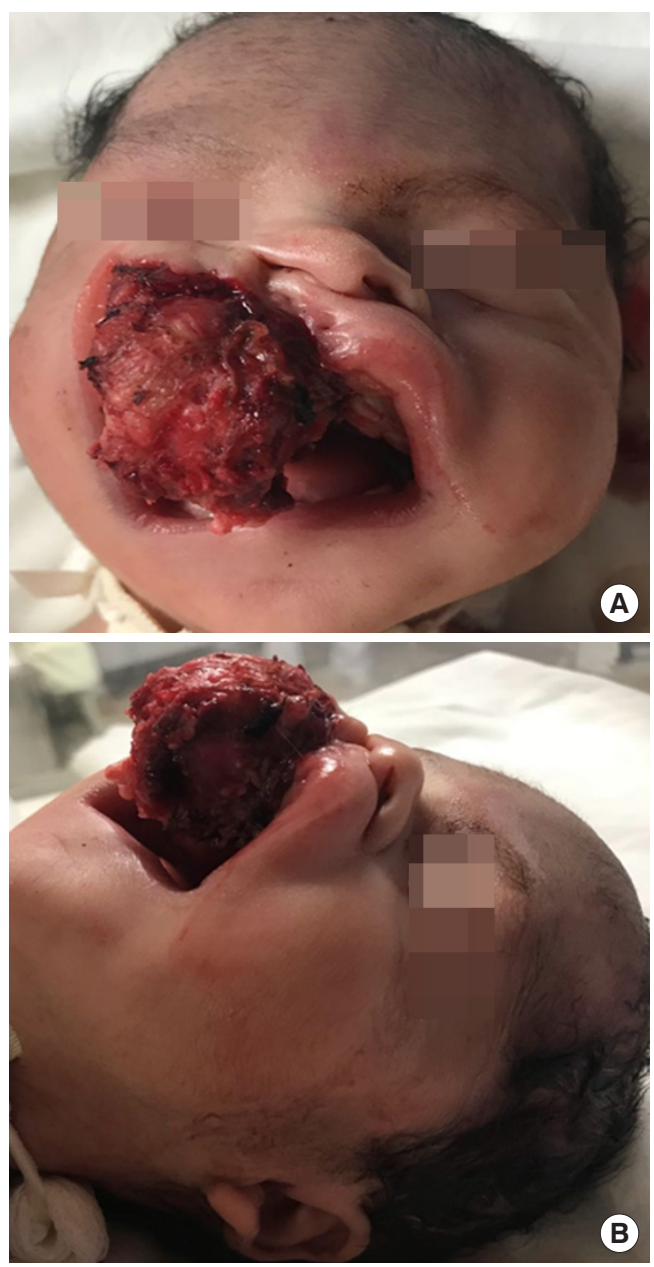

Fig. 2. Residual tumor protruding through the mouth. (A) Frontal and (B) oblique view of the tumor after extraoral resection.

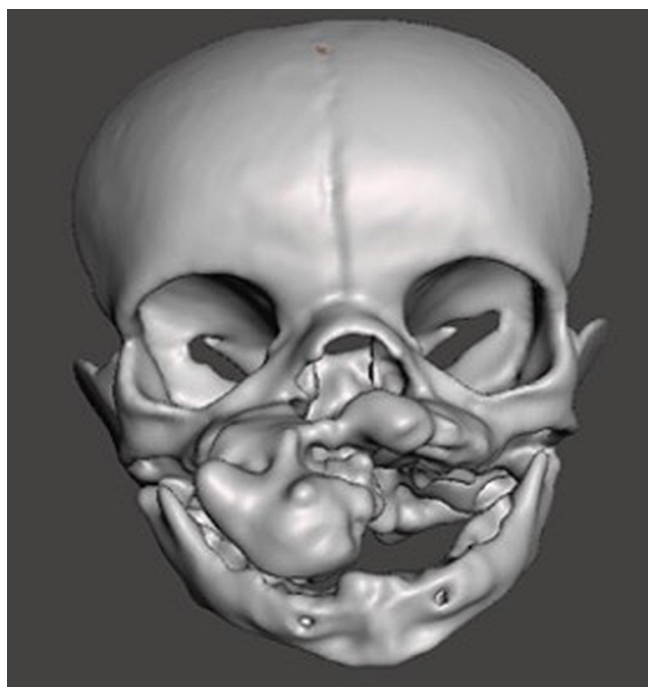

Fig. 3. Computed tomography scan and 3-dimensional (3D) virtual image reconstruction. Frontal view of 3D image reconstruction with the residual tumor occupying the hard palate with extension to the nasopharynx.
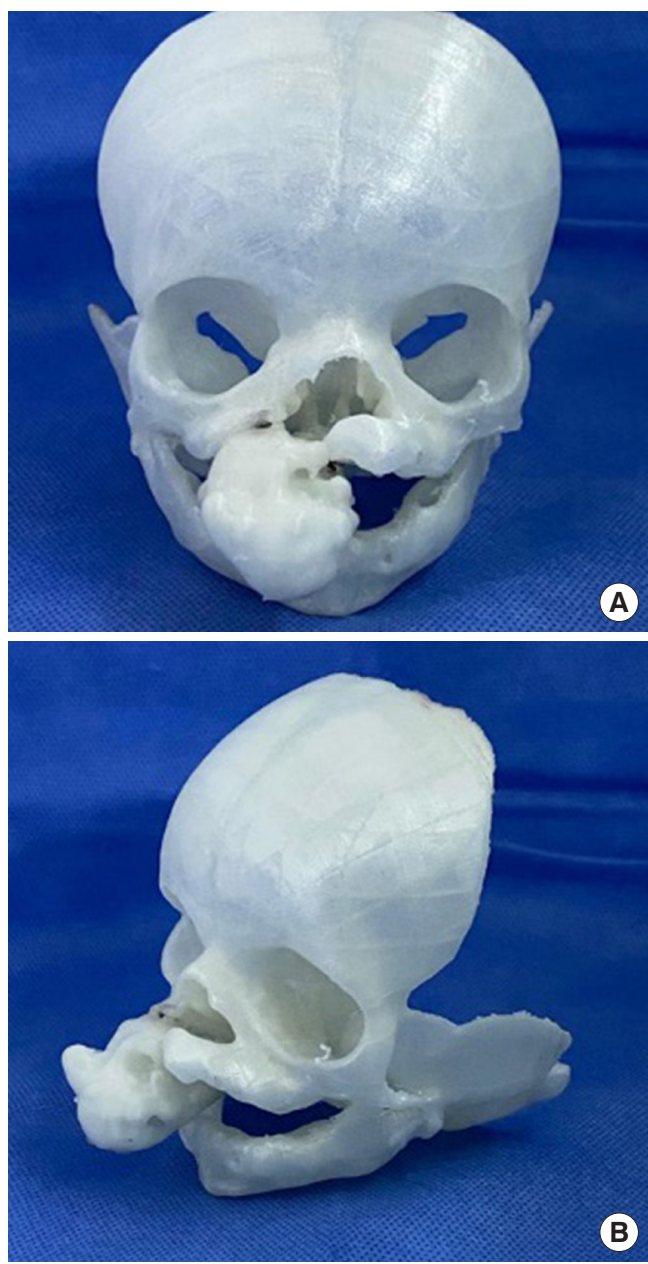

Fig. 4. Stereolithographic 3-dimensional (3D) model of the facial skeleton to scale. (A) Frontal view of the $3 \mathrm{D}$ model and (B) right oblique view of the 3D model. 


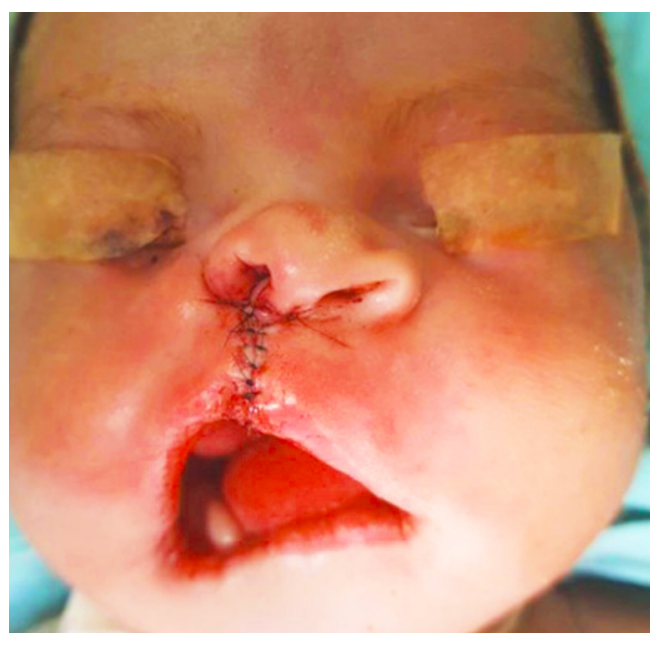

Fig. 5. Intraoperative view of epignathus resection. Final appearance after a bloc resection of the intraoral tumor and lip adhesion.

precisely establish the extension, depth, and relationship of the tumor with surrounding tissues (Fig. 3). Based on this large 3D perspective, we were able to establish exactly the limits of the tumor, and therefore the free millimeter margin to be resected.

As a complement, we manufactured a 3D stereolithographic model of the facial skeleton to scale, which allowed the team members to become more familiar with the surgical act. 3D visualization of the tumor margins was essential, as well as the residual defect that would have to be reconstructed (Fig. 4).

\section{Resection and reconstruction of the epignathus: plastic surgery}

On day 12 after birth, under general anesthesia, a bloc resection of the tumor was performed. This left a palatal soft-tissue defect of $0.7 \times 0.9 \mathrm{~mm}$ and a small bone defect of $0.2 \times 0.3 \mathrm{~mm}$ due to tumor compression on the hard palate; it was possible to cover the defect by a contralateral rotation of a gingival periosteum flap. The great soft-tissue distention caused by the tumor during its growth deformed the anatomy of the cleft of the lip; due to these characteristics, we only perform lip adhesion (Fig. 5). The definitive treatment of the cleft lip and pseudomacrostoma was deferred for a second procedure.

The postoperative period was uneventful; the patient was discharged 10 days after the surgical procedure in agreement with the pediatric surgery service, and subsequent clinical evaluations continued in the outpatient clinic. The following 6 postoperative months passed with adequate healthy-child control. The most predominant aspect was that there was no evidence of tumor recurrence. The gradual regression of tissue distention showed a slight improvement in the appearance of the pseudomacrostoma (Fig. 6). Based on this assessment, it was decided to perform the closure of the cleft of the soft palate. This was

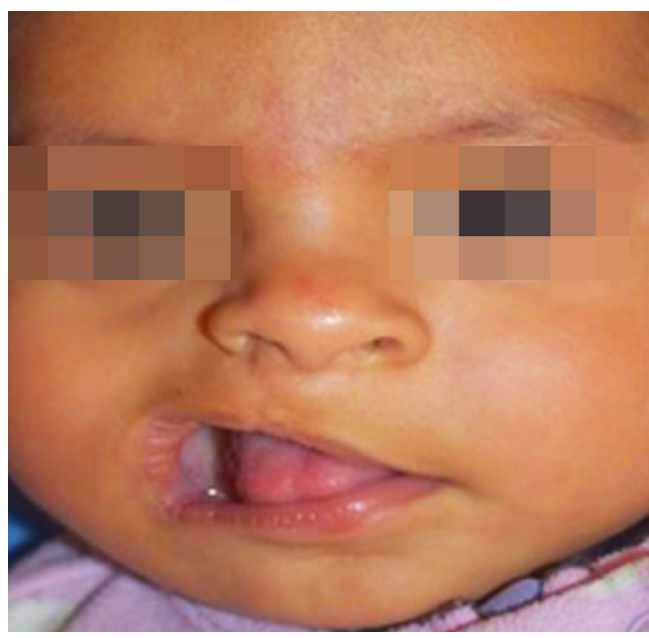

Fig. 6. Postoperative view of the patient. General appearance of the face at 6 months.

carried out without complications; in addition to this, a small touch-up of the primary lip adhesion was also performed, which consisted of an aesthetic refinement of the subunits of the central labial area.

\section{DISCUSSION}

Epignathus is a congenital palatal teratoma. It is an exceedingly rare tumor with an extremely low incidence and it often grows in the prenatal period [8].

This pathology is mostly diagnosed at the end of the second or the third trimester of pregnancy, although 2D ultrasound studies have shown success in detection even from week 15 to 17 of gestation [9]. This could be because prenatal control is inconsistent, or the tumor may develop late in pregnancy [10]. Ultrasound findings of epignathus during a prenatal assessment show a complex mass with cystic and solid components arising from the face or neck combined with polyhydramnios [11].

Several studies have shown that MRI is the best option to obtain greater precision in the prenatal diagnosis, since it offers a better understanding of the anatomical planes and identifies the characteristics and composition of the mass, in addition to providing a broader view of airway and esophageal patency, thus helping to identify fetuses that require an EXIT procedure $[12,13]$.

Kumar et al. [14] described the association between preeclampsia and teratomas, as well as an increase in amniotic alpha-fetoprotein (AFP). In our case, the mother did not suffer from preeclampsia, and no reports of amniotic fluid AFP levels were found because her prenatal control was carried out in an external clinic, and the mother was referred to our hospital after her diagnosis during the third trimester, days before delivery. 
On the second day after birth, abnormally high AFP levels $>1,210 \mu \mathrm{g} / \mathrm{mL}$ were reported compared to a series of reported cases of 260 neonates, in which AFP levels ranged from 15.7 to $146.5 \mu \mathrm{g} / \mathrm{mL}[15]$.

Polyhydramnios is a common complication and occurs in approximately $50 \%$ of cases. This is commonly found in the presence of large tumors because they are associated with a higher probability of tracheal and esophageal occlusion [16]. In our patient, the initial dimensions of the neoplasm were $11.4 \times 9.2 \times 8$ $\mathrm{cm}$ together with the presence of polyhydramnios. Neonatal mortality associated with epignathus is extremely high due to airway obstruction during or shortly after birth [17]. Therefore, the establishment of a safe airway for the neonate is essential if an antenatal tracheal obstruction is suspected.

EXIT was developed to safely and efficiently establish cardiopulmonary support during delivery while maintaining placental gas exchange. It is considered the gold standard for obtaining a safe airway in the fetus with airway obstruction [18]. The procedure is indicated in various pathologies such as severe micrognathia or tracheal anomalies; it is also a viable therapeutic option in the case of oropharyngeal masses.

The process is generally carried out systematically, starting with attempts at endotracheal intubation by direct laryngoscopy, followed by bronchoscopy, and finally, tracheostomy with or without resection of the head or neck tumor; evidently, all procedures are performed under placental support. Complete surgical excision, followed by the repair of associated anomalies as soon as the patient is stabilized, is the standard procedure to treat teratomas [19].

Throughout history, different images have been used to understand, plan, and individualize surgical procedures, and due to innovative advances in current technological tools, 3D models have helped improve crucial steps in craniofacial surgery by providing accurate information on the anatomy of each patient, and in turn, helping to establish surgical planning with a better strategy. The combination of computer-assisted design and model manufacturing (CAD-CAM) has provided efficient models for presurgical planning, increasing the precision required for tissue removal and making it possible to incorporate the best strategy for reconstruction; this without a doubt, translates into a reduction in intraoperative time [20]. These lithographic stereo models can be made based on routine CT or MRI scans and can be converted into a $\mathrm{CAD}$ file using a variety of $3 \mathrm{D}$ software programs. The main strength we had in the use of $3 \mathrm{D}$ printed models was that it allowed us to carry out custom-made preoperative planning, in addition to having a $360^{\circ}$ view of the critical structures adjacent to the tumor. This allowed correct delimitation of the surgical borders for resection, and a more exact view of the defect to be reconstructed. A limitation for the use of 3D models has cost; however, it is expected that with the standardization of its use, the cost of the models will decrease and in turn, cheaper materials will be available.

Because of its low incidence and difficult access, epignathus always represents a challenging surgical situation. The $3 \mathrm{D}$ perspective that virtual images offer, together with the elaboration of 3D models, represents an innovative advance for the comprehensive treatment of this and other types of complex lesions. The strategic planning of the surgical steps favors greater dynamism in the process of observation and synchronous manipulation of tissues. This translates into a higher margin of understanding and safety at the time of performing the resection.

We consider that 3D technology, whether in a printed model, tracings, and/or visualized during the pre and intraoperative phase, has become a novel, practical, and convenient tool for understanding, planning, and managing overly complex cases. The great advantages of using virtual images, together with 3D models, are mainly: a 3D view of the lesion, better definition of surgical borders and interaction with surrounding tissues, and simultaneously having a clear perspective of the resulting defect to incorporate the best reconstruction alternative. We would compare it to conducting "flight simulation." In this case, it is possible to review the surgical steps as many times as necessary before the procedure.

\section{NOTES}

\section{Conflict of interest}

No potential conflict of interest relevant to this article was reported.

\section{Ethical approval}

The study was approved by the Institutional Review Board of University Hospital "Dr. José Eleuterio González", and Faculty of Medicine (IRB No. CP 20-003) and performed in accordance with the principles of the Declaration of Helsinki. Written informed consent was obtained.

\section{Patient consent}

The patient's guardians provided written informed consent for the publication and the use of the patient's images.

\section{Author contribution}

Data curation: PP Flores García, FF Montes-Tapia. Formal analysis: Y Castro-Govea. Methodology: CM Gonzalez-Cantu, Y Castro-Govea. Project administration: CM Gonzalez-Cantu, Y Castro-Govea. Writing - original draft: CM Gonzalez-Cantu, PJ 
Moreno-Peña, MG Salazar-Lara, VH Cervantes-Kardasch, Y Castro-Govea. Writing - review \& editing: Y Castro-Govea.

\section{ORCID}

Cynthia Minerva Gonzalez-Cantu https://orcid.org/0000-0001-7561-1437

Pablo Juan Moreno-Peña

https://orcid.org/0000-0003-3661-4912

Mayela Guadalupe Salazar-Lara https://orcid.org/0000-0002-2710-1528

Pablo Patricio Flores García

https://orcid.org/0000-0003-4891-0604

Fernando Félix Montes-Tapia

https://orcid.org/0000-0003-4407-221X

Victor Hugo Cervantes-Kardasch

https://orcid.org/0000-0002-4844-022X

Yanko Castro-Govea https://orcid.org/0000-0002-1121-4333

\section{REFERENCES}

1. Jadhav SS, Korday CS, Malik S, et al. Epignathus leading to fatal airway obstruction in a neonate. J Clin Diagn Res 2017; 11:SD04-5.

2. Herath M, Siriwardena BSMS, Jayasinghe RD, et al. Oropharyngeal epignathus with partial facial duplication: report of a rare case. Int J Oral Maxillofac Surg 2018;47:442-4.

3. Tunes RS, Cavalcanti GZ, Squarisi JMO, et al. Oral epignathus with maxilla duplication: report of a rare case. Craniomaxillofac Trauma Reconstr 2019; 12:62-6.

4. Kirishima M, Yamada S, Shinya M, et al. An autopsy case of epignathus (immature teratoma of the soft palate) with intracranial extension but without brain invasion: case report and literature review. Diagn Pathol 2018;13:99.

5. ElSherbiny Hamed M, El-Din MHN, Abdelazim IA, et al. Prenatal diagnosis and immediate successful management of isolated fetal epignathus. J Med Ultrasound 2019;27:198201.

6. Bauermeister AJ, Zuriarrain A, Newman MI. Three-dimensional printing in plastic and reconstructive surgery: a systematic review. Ann Plast Surg 2016;77:569-76.

7. Jacobs CA, Lin AY. A new classification of three-dimensional printing technologies: systematic review of three-dimensional printing for patient-specific craniomaxillofacial surgery. Plast Reconstr Surg 2017;139:1211-20.
8. Halterman SM, Igulada KN, Stelnicki EJ. Epignathus: large obstructive teratoma arising from the palate. Cleft Palate Craniofac J 2006;43:244-6.

9. Daskalakis G, Efthimiou T, Pilalis A, et al. Prenatal diagnosis and management of fetal pharyngeal teratoma: a case report and review of the literature. J Clin Ultrasound 2007;35:15963.

10. Tonni G, Centini G, Inaudi P, et al. Prenatal diagnosis of severe epignathus in a twin: case report and review of the literature. Cleft Palate Craniofac J 2010;47:421-5.

11. Kaido Y, Kikuchi A, Oyama R, et al. Prenatal ultrasound and magnetic resonance imaging findings of a hypovascular epignathus with a favorable prognosis. J Med Ultrason (2001) 2013;40:61-4.

12. Kumar KM, Veligandla I, Lakshmi AR, et al. Congenital giant teratoma arising from the hard palate: a rare clinical presentation. J Clin Diagn Res 2016;10:ED03-4.

13. Paula Pinho Matos A, Teixeira Castro P, de Barros Duarte L, et al. Prenatal diagnosis of cervical masses by magnetic resonance imaging and 3D virtual models: perinatal and longterm follow-up outcomes. J Matern Fetal Neonatal Med 2020;33:2181-9.

14. Kumar SY, Shrikrishna U, Shetty J, et al. Epignathus with fetiform features. J Lab Physicians 2011;3:56-8.

15. Kadlub N, Touma J, Leboulanger N, et al. Head and neck teratoma: from diagnosis to treatment. J Craniomaxillofac Surg 2014;42:1598-603.

16. Hodges MM, Crombleholme TM, Marwan AI, et al. Massive facial teratoma managed with the ex utero intrapartum treatment (EXIT) procedure and use of a 3-dimensional printed model for planning of staged debulking. J Pediatr Surg Case Rep 2017; 17:15-9.

17. Bence CM, Wagner AJ. Ex utero intrapartum treatment (EXIT) procedures. Semin Pediatr Surg 2019;28:150820.

18. Hsieh TY, Dedhia R, Cervenka B, et al. 3D printing: current use in facial plastic and reconstructive surgery. Curr Opin Otolaryngol Head Neck Surg 2017;25:291-9.

19. Aldaadaa A, Owji N, Knowles J. Three-dimensional printing in maxillofacial surgery: hype versus reality. J Tissue Eng 2018;9:2041731418770909.

20. Chae MP, Rozen WM, McMenamin PG, et al. Emerging applications of bedside $3 \mathrm{D}$ printing in plastic surgery. Front Surg 2015;2:25. 